Vietnam Journal of Mechanics, NCST of Vietnam Vol. 25, 2003, No 4 (225 - 242)

\title{
THE BALANCING OF ROTATING MACHINERY AS NON-LINEAR SYSTEM PASSING ACROSS A RESONANT REGION
}

\author{
Nguyen Cao Menh and Tran Duong Tri \\ Institute of Mechanics, NCST of Vietnam
}

\begin{abstract}
This paper presents a dynamical balancing method for the rotational part of machine as a nonlinear system with the decrease of rotation speed passing across a resonant region. After analyzing the nonlinear system and measurable vibration signals, a suitable procedure of dynamical balance for determining the magnitude and location of imbalance mass is proposed. A program on PC is made to illustrate the obtained procedure. The results of numeric examples show that it can be used well for dynamical balancing analysis.
\end{abstract}

\section{Introduction}

One of the main causes of machinery vibration is imbalance of rotational part. In special case for rotating machines with high speed, which goes up to ten thousand circles per minute, the rotational parts must be balanced exactly. Two following dynamical balancing methods usually are applied: on-site balance with the fixed rotational speed and on-special balance device with the decreasing rotational circle passing across a resonant region. For a linear system there are some methods to find the magnitude and location of imbalance mass [2]. Nevertheless, for nonlinear systems, those methods have to be investigated more in details to propose suitable procedures [1].

In this paper, a method for calculating imbalance parameters of a nonlinear system has presented. Computational data is used as measurable vibration signals processing in accordance with three-times trial method.

\section{Equations of motion of a rotational part}

\subsection{Equation of motion without a trial mass}

The simplest model of an imbalance rotational part is shown in Fig. 1, in which the rotational angle $\varphi(t)$ is given. The angular velocity and acceleration are defined as $\dot{\varphi}(t), \ddot{\varphi}(t)$.

From the rule of center mass motion, we have

$$
(M+m) \ddot{x}_{c}=\sum F_{x}
$$

where $x_{c}$ is the coordinate of the center mass with respect to the $x$-axis and $\sum F_{x}$ is the sum of the projections of the forces with respect to the $x$-axis. 


$$
x_{c}=\left(M x_{M}+m x_{m}\right) /(M+m) .
$$

Denoting $x_{M}=x$ and use $x$ as the generalized coordinate, we get

$$
\begin{aligned}
& x_{m}=x+r \cos \varphi ; \quad \dot{x}_{m}=\dot{x}-r \dot{\varphi} \sin \varphi ; \quad \ddot{x}_{m}=\ddot{x}-r \dot{\varphi}^{2} \cos \varphi-r \ddot{\varphi} \sin \varphi, \\
& (M+m) \ddot{x}_{c}=M \ddot{x}+m\left(\ddot{x}-r \dot{\varphi}^{2} \cos \varphi-r \ddot{\varphi} \sin \varphi\right) .
\end{aligned}
$$

Assume that the initial position is the equilibrium, the restorative spring force is equal to $G(x)$ and the viscous damping force is proportional to the velocity and is equal to $c \dot{x}$, we have

$$
\sum F_{x}=-G(x)-c \dot{x}
$$

In practice, the function $G(x)$ is odd and the expansion of $G(x)$ is of the form

$$
G(x)=k x+b x^{3}+\ldots
$$

For balancing the rotational part of machinery in state passing across a resonant region, the first, machinery is rotated with a velocity greater than the resonant velocity (approximates twice); the next, the machinery is turned off and rotated free without excitation. When the velocity is passing across the resonant region, the vibration of machine comes great. Because of resistance, the damping forces (friction of pillow-block, air),... always exist; the machinery will rotate and slow down to stop. The angular velocity can be determined by the following expression [1]:

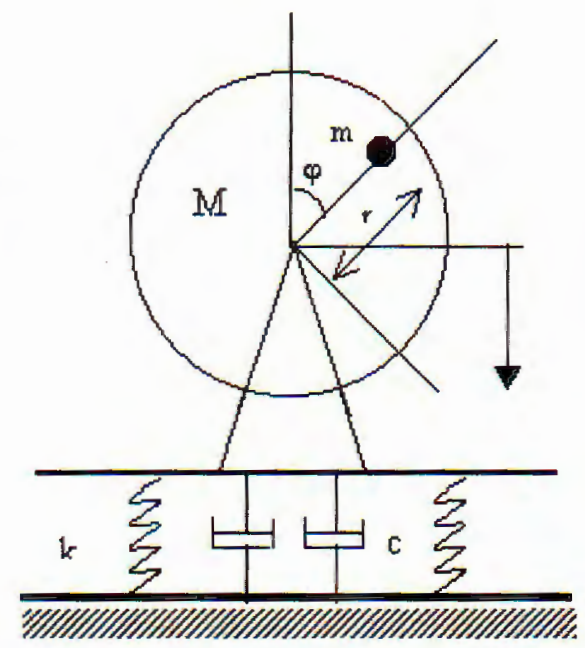

$$
\varphi(t)=\omega\left(1-\frac{\alpha}{2} t\right) t \Rightarrow \dot{\varphi}(t)=\omega(1-\alpha t), \quad \ddot{\varphi}(t)=-\alpha \omega,
$$

where $\omega$ is the angular velocity before it will be decreasing, $\alpha$-coefficient of decreasing rotation. Therefore, the equation of motion of the rotating part of machine (Fig. 2) with the decreasing rotation passing across a resonant region can be written as

$$
\ddot{x}+2 h \dot{x}+\omega_{0}^{2} x+\varepsilon \beta x^{3}=P\left[\dot{\varphi}^{2} \cos \varphi+\ddot{\varphi} \sin \varphi\right],
$$

where: $\quad 2 h=\frac{c}{M+m}, \quad \omega_{0}^{2}=\frac{k}{M+m}, \quad \varepsilon \beta=\frac{b}{M+m}, \quad P=\frac{m r}{M+m}$ $\alpha$-coefficient of decreasing rotation. 


\subsection{Equation of motion with a trial mass}

The model of the rotating part of machine with the trial mass $m_{t}$ is shown in Fig. 2. From the equation of motion of the common mass centre of system, which has the nonlinear spring force is defined with two the first items of expression (2.2), we suppose that the trial mass $m_{t}$ is very small compairing with the mass of rotational body, hence equation of motion with the trial mass $m_{t}$ becomes

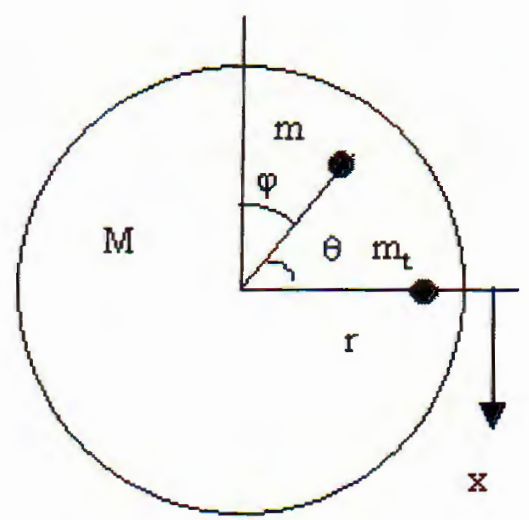

Fig. 2

$$
\ddot{x}+2 h \dot{x}+\omega_{0}^{2} x+\varepsilon \beta x^{3}=P_{1}\left[\dot{\varphi}^{2} \cos \left(\varphi+\phi_{1}\right)+\ddot{\varphi} \sin \left(\varphi+\phi_{1}\right)\right],
$$

where

$$
\begin{aligned}
& \varepsilon \beta=\frac{b}{M+m}, \quad \operatorname{tg} \phi_{1}=\frac{m_{t} \sin \theta}{m+m_{t} \cos \theta}, \quad 0 \leq \phi_{1} \leq \pi \\
& m_{t d 1}=\sqrt{m^{2}+m_{t}^{2}+2 m m_{t} \cos \theta}, \quad P_{1}=\frac{m_{t d 1} r}{M+m}=\frac{m_{t d 1}}{m} P .
\end{aligned}
$$

\subsection{Equation of motion with a radial symmetric trial mass}

Similarly, when the trial mass $m_{t}$ is attached to the rotating part in the place of radial symmetry with respect to old position of $m_{t}$, the respective differential equation is determined by the following expressions:

$$
\ddot{x}+2 h \dot{x}+\omega_{0}^{2} x+\varepsilon \beta x^{3}=P_{2}\left[\dot{\varphi}^{2} \cos \left(\varphi+\phi_{2}\right)+\ddot{\varphi} \sin \left(\varphi+\phi_{2}\right)\right]
$$

where

$$
\begin{aligned}
\operatorname{tg} \phi_{2} & =\frac{-m_{t} \sin \theta}{m-m_{t} \cos \theta}, \quad 0 \leq \phi_{2} \leq \pi \\
m_{t d 2} & =\sqrt{m^{2}+m_{t}^{2}-2 m m_{t} \cos \theta}, \quad P_{2}=\frac{m_{t d 2} r}{M+m}=\frac{m_{t d 2}}{m} P .
\end{aligned}
$$

\section{Determination of balancing parameters}

This section presents the procedure to determine the balancing parameters, after analyzing the solutions of Eqs (2.4), (2.5) and (2.6). From the obtained solutions and the results of processing measurable vibration signal a procedure is proposed for determining the balancing parameters for the dynamic system. The solutions of the nonlinear differential equations, which had been known, using a direct numerical integral method [5] are considered as the measurable vibrations. 


\subsection{A degrading-system}

In Eqs. (2.4), (2.5) and (2.6), putting the nonlinear parameter $\beta=0$, we obtain the respective degrading-equations. A system, which is described by degradingequation, will be called the degrading-system.

\subsubsection{Degrading-equations}

* For rotational part with its imbalances:

$$
\ddot{x}+2 h \dot{x}+\omega_{0}^{2} x=P \omega^{2} F_{0}(t),
$$

where

$$
F_{0}(t)=(1-\alpha t)^{2} \cos \left(\omega\left(1-\frac{\alpha}{2} t\right) t\right)-\frac{\alpha}{\omega} \sin \left(\omega\left(1-\frac{\alpha}{2} t\right) t\right)
$$

* For rotational part with its imbalances and trial mass $m_{t}$ in the first position

$$
\ddot{x}+2 h \dot{x}+\omega_{0}^{2} x=P_{1} \omega^{2} F_{1}(t),
$$

where

$$
F_{1}(t)=(1-\alpha t)^{2} \cos \left(\omega\left(1-\frac{\alpha}{2} t\right) t+\phi_{1}\right)-\frac{\alpha}{\omega} \sin \left(\omega\left(1-\frac{\alpha}{2} t\right) t+\phi_{1}\right)
$$

* For rotational part with its imbalances and trial mass $m_{t}$ in the second position, which is radial symmetric to first one

$$
\ddot{x}+2 h \dot{x}+\omega_{0}^{2} x=P_{2} \omega^{2} F_{2}(t),
$$

where

$$
F_{2}(t)=(1-\alpha t)^{2} \cos \left(\omega\left(1-\frac{\alpha}{2} t\right)+\phi_{2}\right)-\frac{\alpha}{\omega} \sin \left(\omega\left(1-\frac{\alpha}{2} t\right) t+\phi_{2}\right)
$$

The formulae for solutions of Eqs (3.1), (3.2), and (3.3) have very complicated forms $[7,8]$. In this section, a simpler and handier calculative procedure is proposed.

Note 1. From (2.4), (2.5), and (2.6), coefficients $P, P_{1}$ and $P_{2}$ in the expressions representing respective centrifugal forces have the following relations:

$$
P=\frac{m r}{M+m}, \quad P_{1}=\frac{m_{t d 1}}{m} P, \quad P_{2}=\frac{m_{t d 2}}{m} P .
$$

Hence, putting

$$
B D:=\sqrt{\frac{P_{1}^{2}+P_{2}^{2}-2 P^{2}}{2}}
$$

we obtain the formulae to determine the balancing parameters when $P, P_{1}, P_{2}$, and $m_{t}$ are given:

$$
m=P \frac{m_{t}}{B D}, \quad \theta=\arccos \left(\frac{P_{1}^{2}-P_{2}^{2}}{4 P * B D}\right)
$$




\subsubsection{The solutions of degrading-systems}

* In the case of degrade-system without the trial mass $m_{t}$

The initial conditions of Eq. (3.1) are chosen from its stationary solutions putting $\alpha=0$. Therefore, we have

$$
x(0)=\frac{P \omega^{2}}{\sqrt{\left(\omega_{0}^{2}-\omega^{2}\right)^{2}+4 h^{2} \omega^{2}}} ; \quad \dot{x}(0)=0 .
$$

Next, the general solution of Eq. (3.1) with condition (3.7) is the form [9]:

$$
\begin{aligned}
x(t) & =e^{-h t}\left[x(0) \cos \lambda t+\frac{\dot{x}(0)+h x(0)}{\lambda} \sin \lambda t\right]+\frac{1}{\lambda} \int_{0}^{t} e^{-h(t-\tau)} \sin \lambda(t-\tau) F_{0}(\tau) d \tau, \\
\lambda^{2} & =\omega_{0}^{2}-h^{2}, \quad \omega \gg h .
\end{aligned}
$$

The integral in expression (3.8) cannot be expressed by elemental functions. We propose the approximate formulae as follows:

Interval $[0, t]$ is divided by. $\tau_{k-1}=(k-1) \Delta \tau \quad k=1,2, \ldots, N+1$, and $\Delta \tau=\frac{t}{N}$.

Function $F_{0}(\tau)$ is approximated by the following function:

$$
F_{0}(\tau)=F_{0}\left(\tau_{k-1}\right) \quad \text { when } \quad \tau_{k-1} \leq \tau \leq \tau_{k} \quad k=1,2, \ldots, N+1 .
$$

Putting

$$
H_{0}:=\frac{1}{\sqrt{\left(\omega_{0}^{2}-\omega^{2}\right)^{2}+4 h^{2} \omega^{2}}}, \quad \operatorname{tg} \psi_{0}:=\frac{\lambda}{h} .
$$

After some uncomplicated calculates, the solution (3.8) is determined by:

$$
\begin{aligned}
x(t) & =P \omega^{2} e^{-h t}\left\{\left(H_{0}-\frac{D}{\lambda \omega_{0}}\right) \cos \lambda t+\left(\frac{h H_{0}}{\lambda}+\frac{C}{\lambda \omega_{0}}\right) \sin \lambda t\right\}, \\
C & :=\sum_{k=1}^{N+1} F_{0}\left(\tau_{k-1}\right) e^{h \tau_{k-1}}\left\{e^{\frac{h t}{N}} \cos \left(\lambda \tau_{k}-\psi_{0}\right)-\cos \left(\lambda \tau_{k-1}-\psi_{0}\right)\right\}, \\
D & :=\sum_{k=1}^{N+1} F_{0}\left(\tau_{k-1}\right) e^{h \tau_{k-1}}\left\{e^{\frac{h t}{N}} \sin \left(\lambda \tau_{k}-\psi_{0}\right)-\sin \left(\lambda \tau_{k-1}-\psi_{0}\right)\right\} .
\end{aligned}
$$

* In the case of degrading-system with the trial mass $m_{t}$

The solution of Eq. (3.2) is of the form:

$$
\begin{aligned}
x_{1}(t) & =P_{1} \omega^{2} e^{-h t}\left\{\left(H_{0}-\frac{D_{1}}{\lambda \omega_{0}}\right) \cos \lambda t+\left(\frac{h H_{0}}{\lambda}+\frac{C_{1}}{\lambda \omega_{0}}\right) \sin \lambda t\right\}, \\
C_{1} & :=\sum_{k=1}^{N+1} F_{1}\left(\tau_{k-1}\right) e^{h \tau_{k-1}}\left\{e^{\frac{h t}{N}} \cos \left(\lambda \tau_{k}-\psi_{0}\right)-\cos \left(\lambda \tau_{k-1}-\psi_{0}\right)\right\}, \\
D_{1} & :=\sum_{k=1}^{N+1} F_{1}\left(\tau_{k-1}\right) e^{h \tau_{k-1}}\left\{e^{\frac{h t}{N}} \sin \left(\lambda \tau_{k}-\psi_{0}\right)-\sin \left(\lambda \tau_{k-1}-\psi_{0}\right)\right\} .
\end{aligned}
$$


* In the case of degrading-system with the radial symmetric trial mass $m_{t}$ The solution of Eq. (3.3) is determined by

$$
\begin{aligned}
x_{2}(t) & =P_{2} \omega^{2} e^{-h t}\left\{\left(H_{0}-\frac{D_{2}}{\lambda \omega_{0}}\right) \cos \lambda t+\left(\frac{h H_{0}}{\lambda}+\frac{C_{2}}{\lambda \omega_{0}}\right) \sin \lambda t\right\}, \\
C_{2} & :=\sum_{k=1}^{N+1} F_{2}\left(\tau_{k-1}\right) e^{h \tau_{k-1}}\left\{e^{\frac{h t}{N}} \cos \left(\lambda \tau_{k}-\psi_{0}\right)-\cos \left(\lambda \tau_{k-1}-\psi_{0}\right)\right\}, \\
D_{2} & :=\sum_{k=1}^{N+1} F_{2}\left(\tau_{k-1}\right) e^{h \tau_{k-1}}\left\{e^{\frac{h t}{N}} \sin \left(\lambda \tau_{k}-\psi_{0}\right)-\sin \left(\lambda \tau_{k-1}-\psi_{0}\right)\right\} .
\end{aligned}
$$

\subsubsection{Calculating the balancing parameters of degrading-systems}

We can rewrite the solutions (3.9), (3.10), and (3.11) in the forms:

$$
x(t)=P \omega^{2} H(t), \quad x_{1}(t)=P_{1} \omega^{2} H_{1}(t), \quad x_{2}(t)=P_{2} \omega^{2} H_{2}(t) .
$$

According to the three-times trial method, the rotor of machine is forced to rotate three times with the same angular velocity $\omega\left(\omega>\omega_{0}, \omega_{0}\right.$ is the resonant frequency), then the rotor is rotated freely without electricity. The measurable vibration results are obtained in the forms of records:

$$
\begin{aligned}
& x\left(t_{k}\right), x_{1}\left(t_{k}\right), x_{2}\left(t_{k}\right): t_{0}=0, t_{N}=T=\frac{1}{\alpha}, \\
& \Delta t=T /(N-1), \quad t_{k}=t_{0}+(k-1) \Delta t, \quad k=1,2, \ldots, N ;
\end{aligned}
$$

where the passing interval of time $[0, T]$ is calculated from time, at which the rotor is beginning rotation with the initial velocity $\omega$, and then it is freely rotated until the rotation is stopped. Rotational resistant coefficient $\alpha$ will have been computed in measurement process.

Responses $x, x_{1}, x_{2}$ have the respective maximum amplitudes at the same times $t_{R}, x^{\max }, x_{1}^{\max }, x_{2}^{\max }$, and then from Eq. (3.12) we obtain:

$$
x^{\max }=P \omega H^{\max }, \quad x_{1}^{\max }=P_{1} \omega^{2} H_{1}^{\max }, \quad x_{2}^{\max }=P_{2} \omega^{2} H_{2}^{\max } .
$$

We can check that (see Table 1)

$$
H^{\max } \approx H_{1}^{\max } \approx H_{2}^{\max } .
$$

Then:

$$
P=\frac{x^{\max }}{\omega^{2} H^{\max }}, \quad P_{1}=\frac{x_{1}^{\max }}{\omega^{2} H^{\max }}, \quad P_{2}=\frac{x_{2}^{\max }}{\omega^{2} H^{\max }} .
$$

Formula (3.16) for (3.4), (3.5), (3.6) allows us to determine the balancing parameters from the three-times trial process of degrading-system in a transient stage. 
Note 2. In practice, formulae (3.4), (3.5), and (3.6) are replaced by the following:

$$
\begin{aligned}
& B D^{\text {test }}:=\sqrt{\frac{\left(x_{1}^{\max }\right)^{2}+\left(x_{2}^{\max }\right)^{2}-2\left(x^{\max }\right)^{2}}{2}}, \\
& m=x^{\max } \frac{m_{t}}{B D^{\text {test }}}, \quad \theta=\arccos \left(\frac{\left(x_{1}^{\max }\right)^{2}-\left(x_{2}^{\max }\right)^{2}}{4 x^{\text {max }} * B D^{\text {test }}}\right) .
\end{aligned}
$$

\subsubsection{Determination of parameters of degrading-system from vibration records}

For above degrading-system, the parameters of system are damping coefficient, natural frequencies, coefficient of decreasing, ...they can be determined from vibration records in the following order:

1. Calculate times, at which the vibration amplitude is maximum, $t_{R}$;

2. Analyze the amplitude spectral to calculate the natural frequency with respect to the maximum amplitude spectral [4];

3. Analyze vibration to calculate damping coefficient $h$, coefficient of decreasing $\lambda[3,9]$.

\subsubsection{Numerical results}

The programs on PC had been created to do the following main functions:

* Create test vibration records: measurement data are obtained by mean of direct solving the differential equations, which describe the vibrations of rotational part, using the numerical methods (Runge-Kutta (4,5), DormandPrince; Runge-Kutta (2,3), Bogacki and Shampine; or formula Adams-BashforthMoulton) [5].

* Calculate the parameters of system (particular frequencies, damping coefficient, deadening frequency, ...) from the obtained vibration records.

* Calculate the balancing parameters.

Example 3.1. The first, the characteristic parameters of vibration system are given. The values $x(t), x_{1}(t), x_{2}(t)$, will be obtained by a direct integral method applying for the differential equations of motion. Those solutions are considered respective the same as the results of vibration measurement in the cases: without trial mass, with trial mass and with radial symmetric trial mass. The solutions of degrading-system from established analytic model (SAM) are shown in Fig. 3. From the graphs we obtain that solutions $x(t), x_{1}(t), x_{2}(t)$ have the same time, at which amplitudes achieve the maximum values. At the same time, resonant amplitudes occur at excited frequencies, which are less than respective particular frequencies. After the resonant time, vibrations will deaden $[7,8]$. 


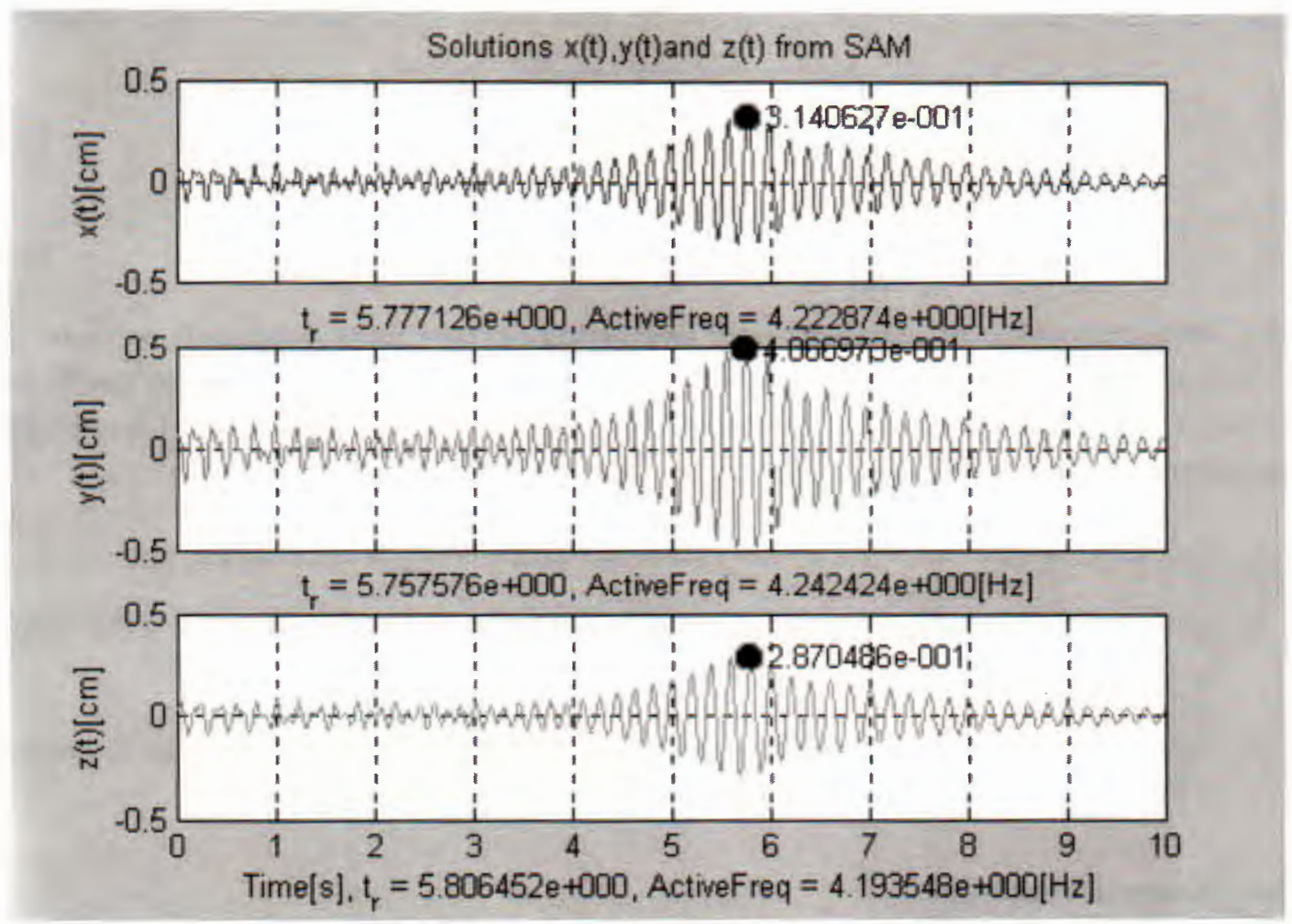

Fig. 3.The solutions of degrade-system from established analytic model (SAM)

Table 1 is the numerical results for description of the vibrations of algorithm model. Fig. 4 shows the amplitude changes of responses $x(t), x_{1}(t), x_{2}(t)$ along with time. We find the same time, at which the amplitudes are maximums. After the first maximum values, the amplitude of vibration and the maximum values will decrease $[7,8]$.

The graphs of functions $H(t), H_{1}(t), H_{2}(t)$ from formula (3.12) are shown in Fig. 5. It is clear that the functions have the same time, at which $H(t), H_{1}(t), H_{2}(t)$ will achieve maximum values. Those maximum values are approximately equal each other. Table 2 is the Test-dynamical parameters of algorithm model. Table 3 is assumed/Calculated imbalance parameters from computational models.

The approximation of the results shows that the above algorithm can be used well.

\section{The characteristic parameters of vibration-system}

The mass of rotational part, $M=2000 \mathrm{~kg}$; Trial mass, $m_{t}=0.8 \mathrm{~kg}$; Rotational radius, $r=50 \mathrm{~cm}$; The initial forced frequency, $\omega=2 \pi f, f=12 \mathrm{~Hz}(\omega=720$ circular per minute); The particular frequency of system, $\omega_{0}=2 \pi f_{0}, f_{0}=5 \mathrm{~Hz}$ $\left(\omega_{0}=300 \mathrm{cir} / \mathrm{min}\right)$; Rotational resistant coefficient $\alpha=0.02 ; 0.04 ; 0.06 ; 0.08 ; 0.10$; $0.12 ; 0.14 ; 0.16 ; 0.18 ; 0.20$, Damping coefficient $h=0.5$ 


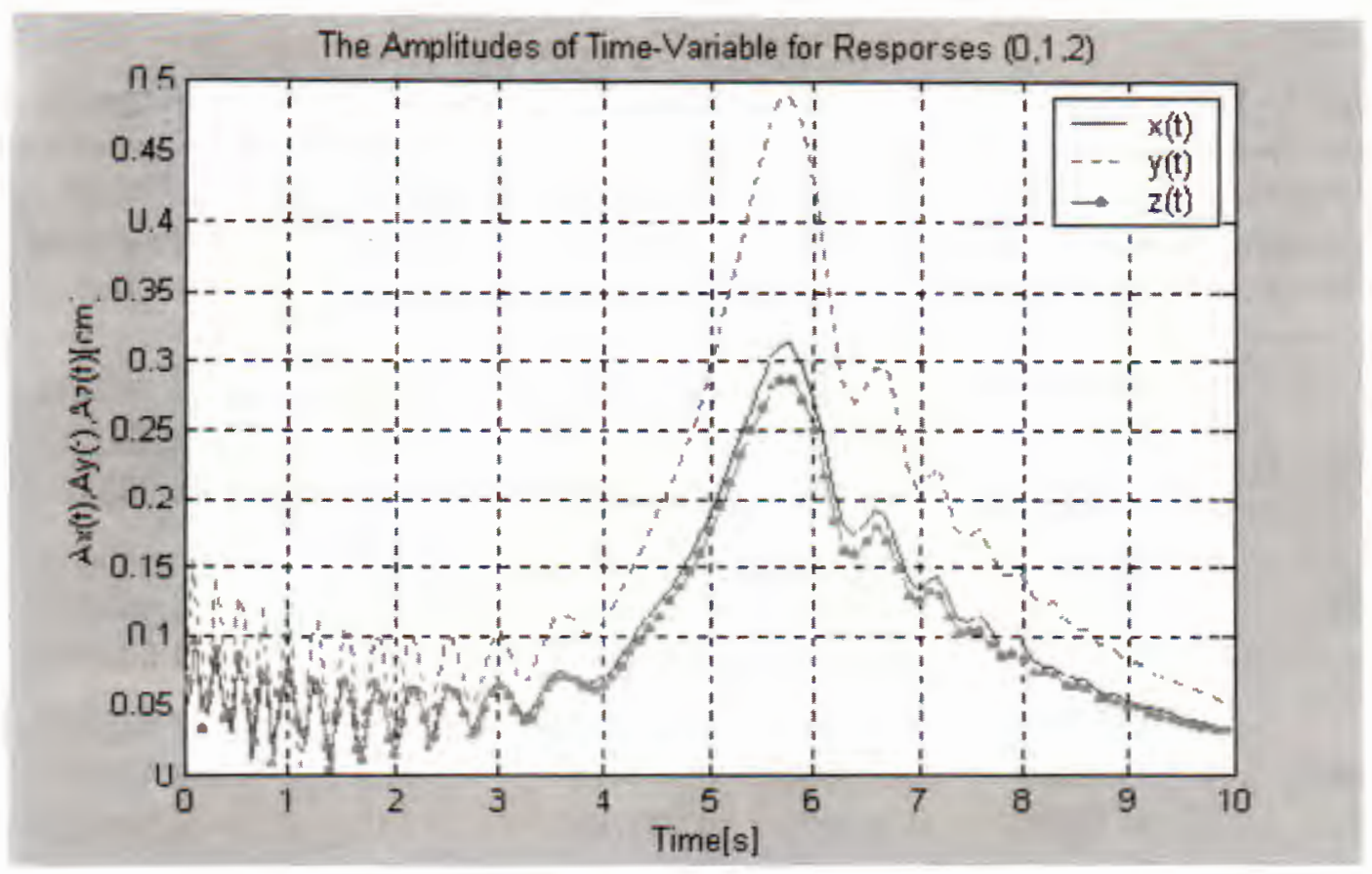

Fig. 4. The amplitude of time of responses
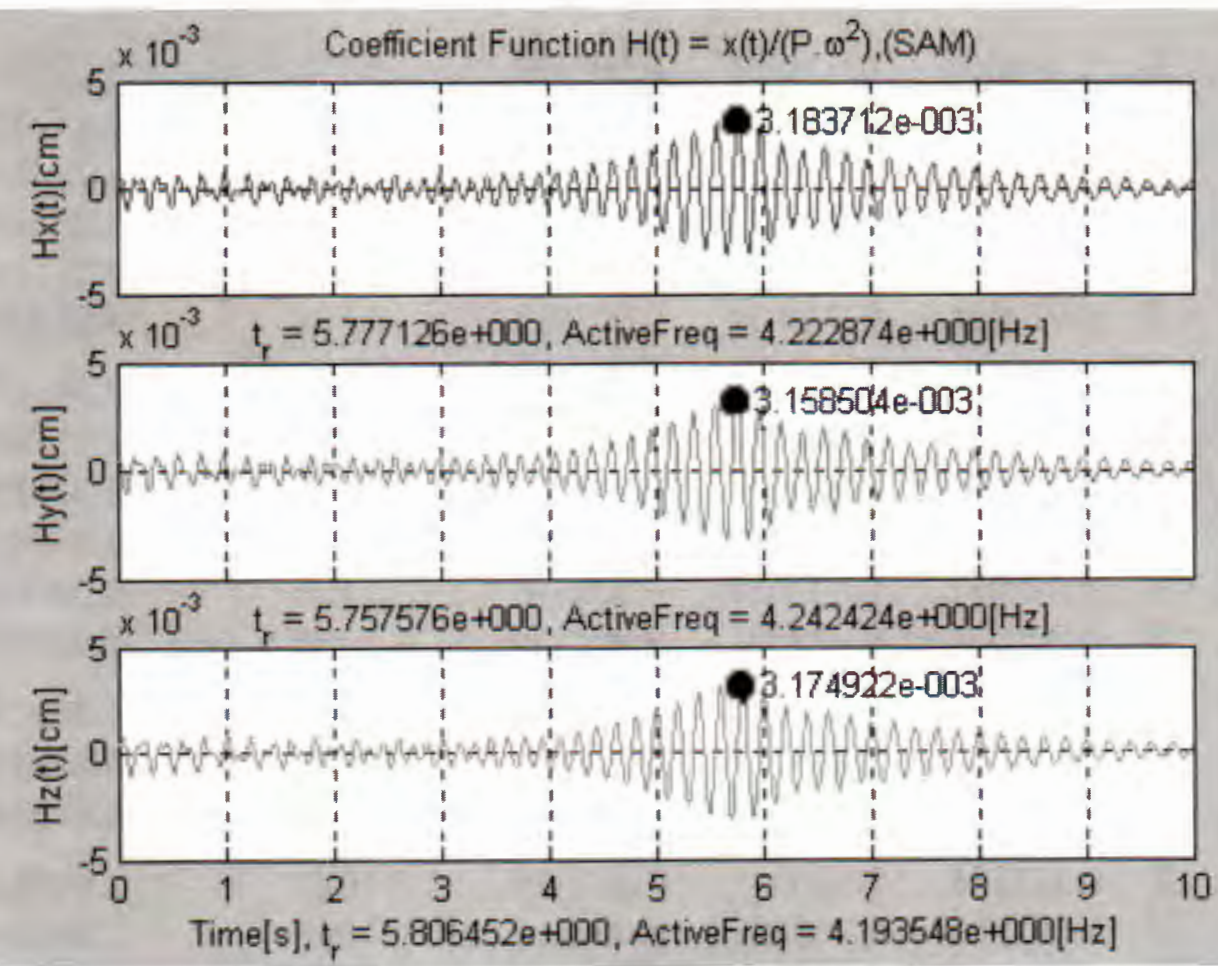

Fig. 5. Coefficient functions $H(t), H_{1}(t)$ and $H_{2}(t)$ 
Table 1. The numerical results for description of the vibrations of algorithm model

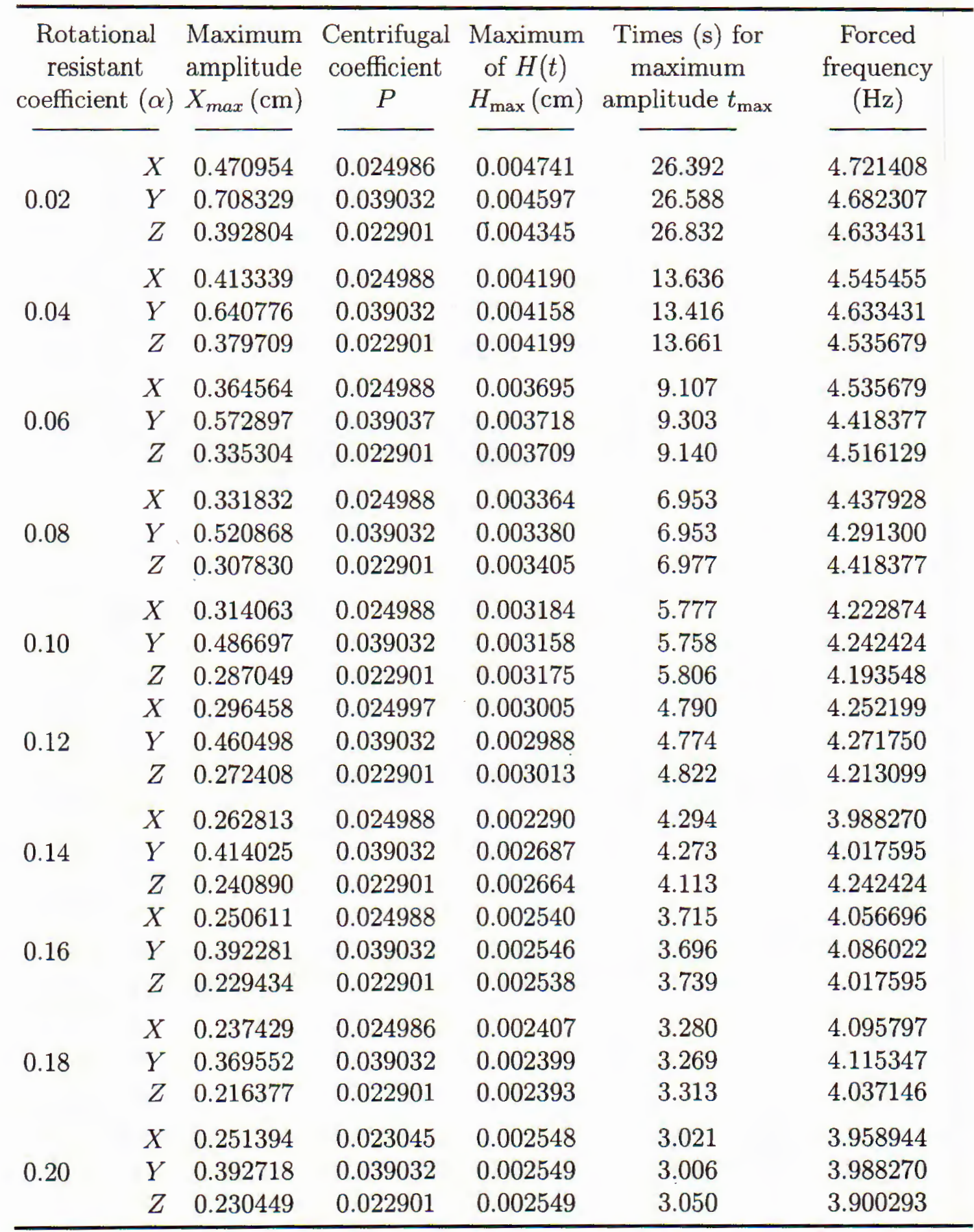


Table 2. The Test-dynamical parameters of algorithm model

\begin{tabular}{|c|c|c|c|c|c|c|}
\hline \multirow{2}{*}{$\begin{array}{c}\text { Rotational } \\
\text { resistant } \\
\text { coefficient } \\
\alpha\end{array}$} & \multicolumn{2}{|c|}{$\begin{array}{c}\text { Damping coefficient } \\
h\end{array}$} & \multirow{2}{*}{$\begin{array}{c}\text { Relative } \\
\text { error } \\
{[\%]}\end{array}$} & \multicolumn{2}{|c|}{$\begin{array}{c}\text { Particular Frequency } \omega_{c} \\
{[\mathrm{rad} / \mathrm{s}]}\end{array}$} & \multirow{2}{*}{$\begin{array}{c}\text { Relative } \\
\text { error } \\
{[\%]}\end{array}$} \\
\hline & Assumed & Calc & & Assumed & & \\
\hline 0 & 00000 & 0. & 3.6 & 31.41593 & & 0.0 \\
\hline & - & & & - & & \\
\hline & - & & & - & & \\
\hline & - & & & - & & \\
\hline & - & & 9 & - & & 35 \\
\hline & - & & 6 & - & & 32474 \\
\hline & - & & & - & & 35041 \\
\hline & - & 1 & 11.821374 & - & & 092278 \\
\hline 0.20 & - & 0.548317 & 8.811894 & - & 31.45529 & 0.125154 \\
\hline
\end{tabular}

Table 3. Assumed/Calculated imbalance parameters from computational models

\begin{tabular}{|c|c|c|c|c|}
\hline \multirow{2}{*}{$\begin{array}{l}\text { Rotational resistant } \\
\text { coefficient } \\
(\alpha)\end{array}$} & \multicolumn{2}{|c|}{ Imbalance mass $m(\mathrm{~kg})$} & \multicolumn{2}{|c|}{ Angle $\theta$ (radian) } \\
\hline & Assumed & Calculated & Assumed & Calculated \\
\hline 0.02 & 1.00000 & 1.156047 & 1.04720 & 0.969267 \\
\hline 0.04 & - & 1.013082 & - & 1.054486 \\
\hline 0.06 & - & 0.986449 & - & 1.046633 \\
\hline 0.08 & - & 0.983082 & - & 1.055771 \\
\hline 0.10 & - & 1.017281 & - & 1.049646 \\
\hline 0.12 & - & 1.009041 & - & 1.053433 \\
\hline 0.14 & - & 0.984024 & - & 1.041626 \\
\hline 0.16 & - & 0.996775 & - & 1.044731 \\
\hline 0.18 & - & 1.010664 & - & 1.043925 \\
\hline 0.20 & - & 0.999745 & - & 1.047332 \\
\hline
\end{tabular}

\section{Assumed parameters}

Imbalance mass, $m=1 \mathrm{~kg}$; Locative angle of imbalance mass, $\theta=\pi / 3 \approx$ 1.047198; The number of times, $N=1024$; The number of times for the approximation of integral, Nint $=500$; The above solutions are considered as the functions of time, $t \in[0, T]$, for $T=1 / \alpha$, where $\alpha$ is the rotational resistant coefficient, which is given $\alpha=0.10$. 
Table 1 shows that:

* If rotational resistant coefficient increases then vibration amplitude will decrease; The time, at which amplitude has the maximum value, will decrease; Resonance frequency, in general, will decrease. In the all calculated cases, the resonance frequency always is less than the particular frequency $f_{0}=\omega_{0} / 2 \pi=$ $5.0 \mathrm{~Hz}$.

* If rotational resistant coefficient is fixed, the time for the maximum amplitudes approximate the same (approximately one tenths second); The maximum value of functions $H(t)(\mathrm{cm})$, coincides to the third decimal number, therefore they can be considered the same. i.e. the supposition (3.15) is satisfied. This is very important in practice.

Table 2 shows that, calculating the test-dynamic parameters of algorithm model $\left(h, \omega_{0}\right)$, for the change of rotational resistant coefficient $\alpha \in[0.04: 0.02: 0.20]$ from measurable records of vibrations:

* For damping coefficient $h$, the minimum relative error is $3 \%$ and maximum one is $11 \%$

* For the particular frequency $\omega_{0}$, the minimum relative error is $0.01 \%$ and maximum one is $0.1 \%$

\section{Three times trial procedure for degrading-system}

Step 1. Input data creating

First Times: The rotational machinery is rotated forcedly with the initial angular velocity $\omega>\omega_{0}$ and then it will be rotated freely.

* Measure the vibration as function of time of the rotational part, $x(t)$;

* Determine the maximum amplitude of measured vibration, $x_{\max }$.

Second Times: The trial mass $m_{t}$ is added at distance $r$ from shaft line. Next, rotational part is rotated with the angular velocity $\omega>\omega_{0}$ then it will be rotated freely.

* Measure the vibration of the rotational part: $x_{1}(t)$;

* Determine the maximum amplitude of vibration $x_{1 \text { max }}$.

Third Times: Adding the trial mass $m_{t}$ at the radial symmetric place of the rotational part, and it is rotated with the angular velocity $\omega$, then will be rotated freely.

* Measure the vibration of the rotational part: $x_{2}(t)$;

* Determine the maximum amplitude of vibration $x_{2 \max }$.

Step 2. Determination of balancing parameters

* Calculate mass $m$, balancing place $\theta$ from formulae (3.17) and (3.18). 
* Calculate $P, P_{1}, P_{2}$ from formulae (2.4), (2.5), and (2.6).

* Calculate $H=x_{\max } / P, H_{1}=x_{1 \max } / P_{1}$ and $H_{2}=x_{2 \max } / P_{2}$ and if $H \approx H_{1} \approx$ $\mathrm{H}_{2}$ then the obtained balancing parameters will have been accepted.

\subsection{Vibration analyzing for weakly non-system}

The solutions of the weakly nonlinear systems (2.4), (2.5), and (2.6) can be considered the same as the measurable vibration records (3.13). In the above equation of motion, nonlinear function $f(x)=x^{3}$ is approximated by a linear function using the minimum square method [6], in which the experimental values of functions are the vibration records in expression (3.13). Therefore, the weakly non-linear systems $(2.4),(2.5)$ and (2.6) had been replaced by the respective degrading-systems, and then the above analytic methods would have been used.

\subsubsection{Approximate systems}

Denoting $x_{j}=x\left(t_{j}\right), x_{j 1}=x_{1}\left(t_{j}\right), x_{j 2}=x_{2}\left(t_{j}\right)$ in expression (3.3), the non-linear systems (2.4), (2.5), and (2.6) become:

* System without the trial mass $m_{t}$

$$
\left\{\begin{array}{l}
\ddot{x}+2 h \dot{x}+\left(\omega_{0}^{2}+\varepsilon \beta \xi\right) x=P \omega^{2} F_{0}(t) \\
\xi=\sum_{j=1}^{N} x_{j}^{4} / \sum_{j=1}^{N} x_{j}^{2}
\end{array}\right.
$$

* System attaching to the trial mass $m_{t}$

$$
\left\{\begin{array}{l}
\ddot{x}+2 h \dot{x}+\left(\omega_{0}^{2}+\varepsilon \beta \xi_{1}\right) x=P_{1} \omega^{2} F_{1}(t) \\
\xi_{1}=\sum_{j=1}^{N} x_{j 1}^{4} / \sum_{j=1}^{N} x_{j 1}^{2}
\end{array}\right.
$$

* System attaching to the radial symmetric trial mass $m_{t}$

$$
\left\{\begin{array}{l}
\ddot{x}+2 h \dot{x}+\left(\omega_{0}^{2}+\varepsilon \beta \xi_{2}\right) x=P_{2} \omega^{2} F_{2}(t) \\
\xi_{2}=\sum_{j=1}^{N} x_{j 2}^{4} / \sum_{j=1}^{N} x_{j 2}^{2}
\end{array}\right.
$$

\subsubsection{The solutions of direct problem}

Assume that, the dynamic characteristic parameters of system $\left(h, \omega_{0}, \omega, \alpha, P, \ldots\right)$ are given we constitute measurable data applying the above numerical integral methods to find the solutions of nonlinear differential Eqs. (2.4), (2.5), and (2.6).

We choose the initial conditions from the stationary solutions of respective nonlinear systems when the forcing functions have the rotational resistant coefficients are equal to zero. 
For example, from system (2.4):

$$
\ddot{x}+2 h \dot{x}+\omega_{0}^{2} x+\varepsilon \beta x^{3}=P\left[\dot{\varphi}^{2} \cos \varphi+\ddot{\varphi} \sin \varphi\right]
$$

the initial conditions are chosen from the stationary solutions of the following equation

$$
\begin{aligned}
& \ddot{x}+2 h \dot{x}+\omega_{0}^{2} x+\varepsilon \beta x^{3}=P \omega^{2} \cos \omega t \\
& x(0)=0, \quad \dot{x}(0)=0
\end{aligned}
$$

The numerical solutions are obtained by approximations (3.19), (3.20), and (3.21) in formulae (3.9), (3.10), and (3.11) - LAM.

Example 3.2. The parameters are given the same ones in example 3.1, nonlinear parameter $\beta=0.5$. A program on PC, had been built by us, leads to the graphs: of the solutions and the amplitude spectra of responses in Figs 6, 7. From the graph of amplitude spectra, Fig. 8, the particular frequencies are the same. This fact shows that the small nonlinear coefficient have very small effect on the particular frequency of system.

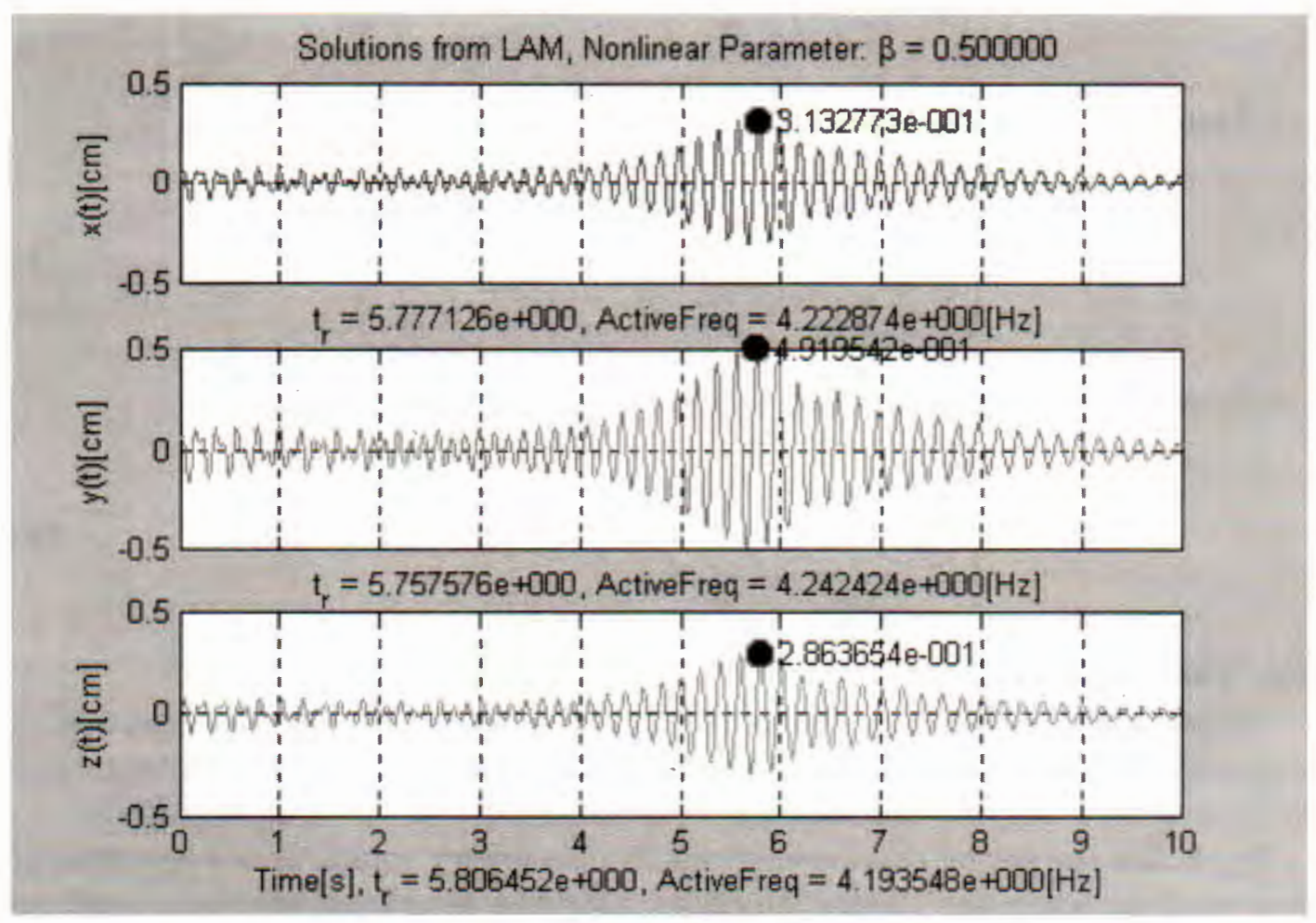

Fig. 6.The solutions of nonlinear system using the linear approximate method (LAM) 

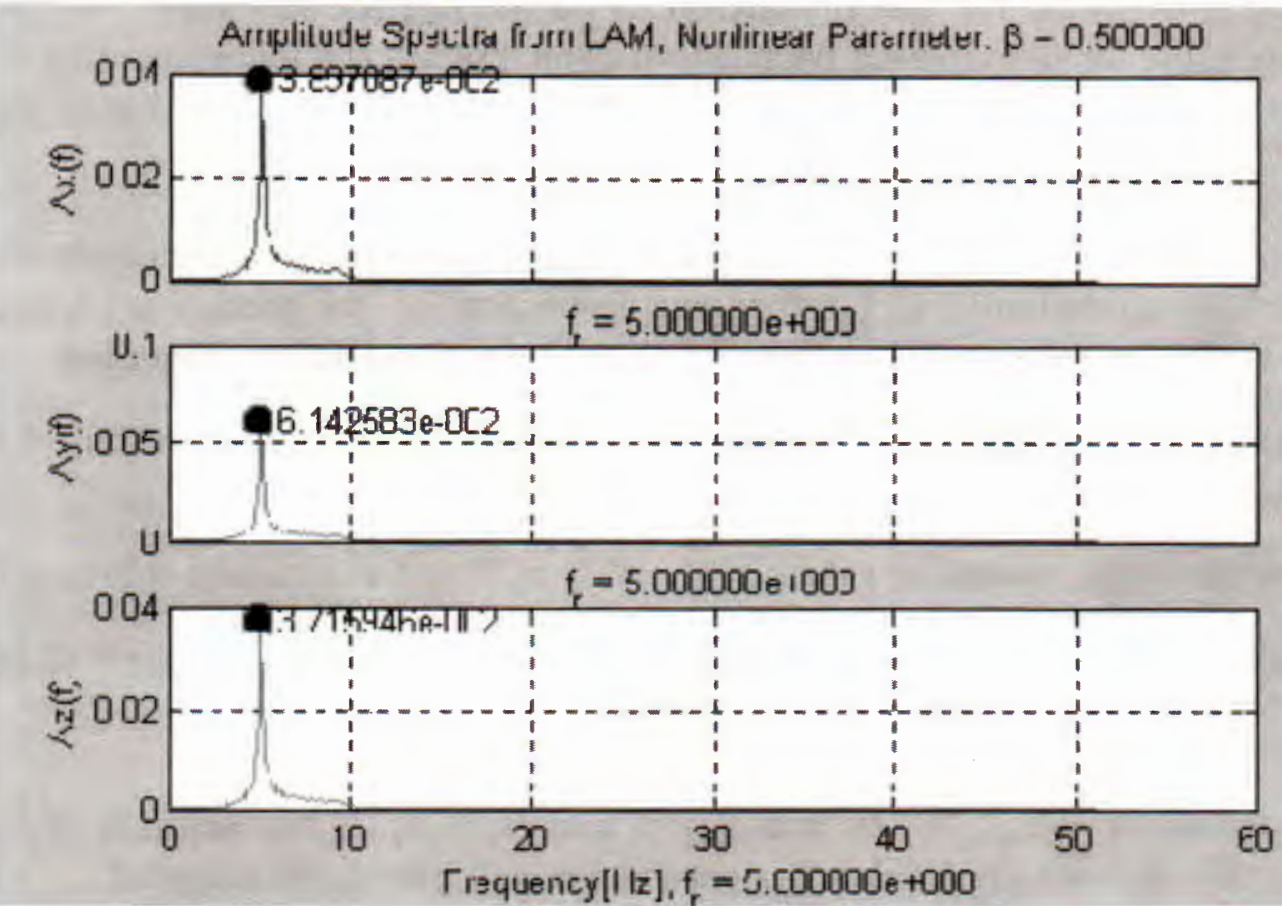

Fig. 7. The amplitude spectra of responses (LAM)

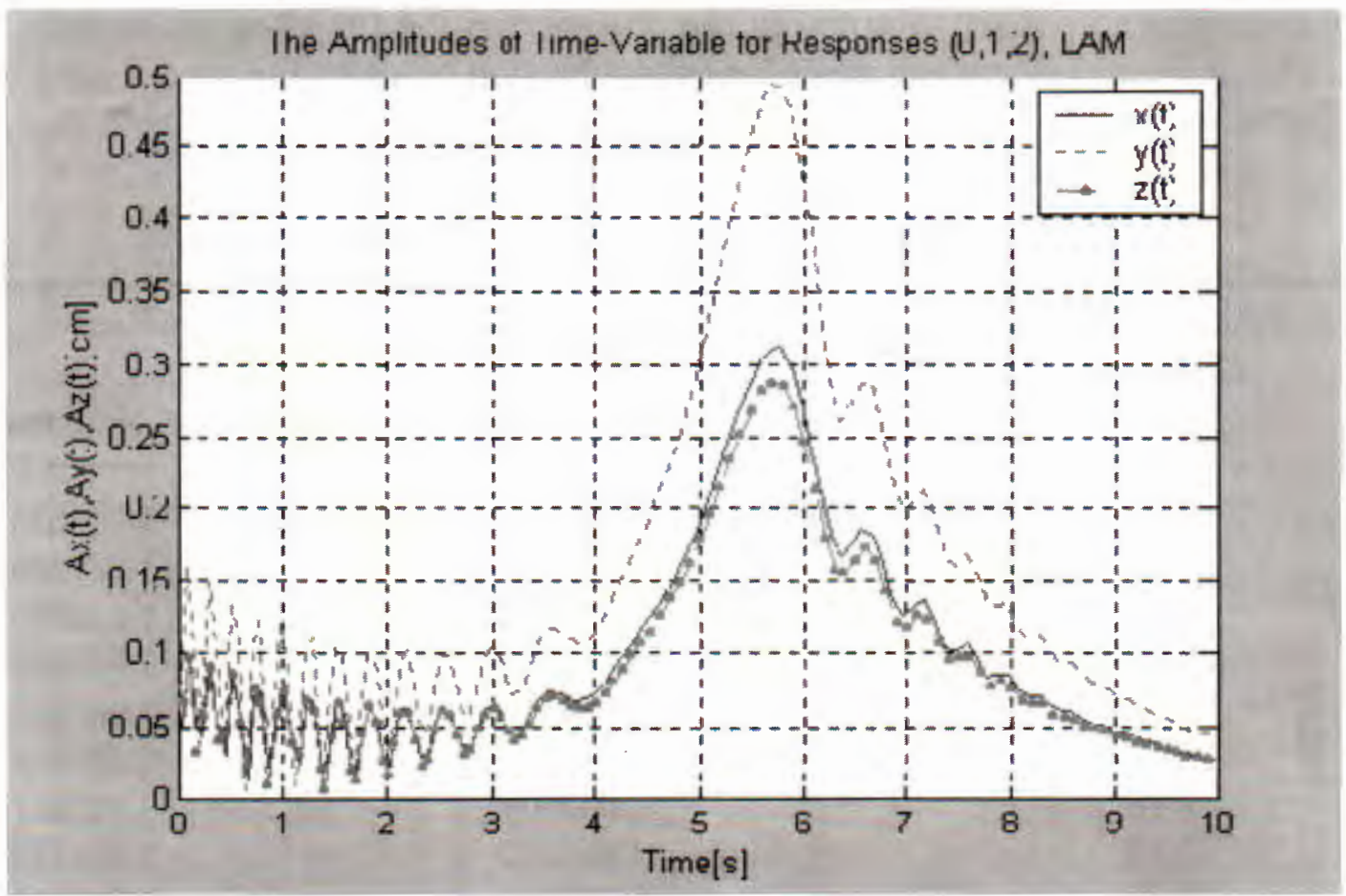

Fig. 8. Amplitudes belong of time of responses, LAM 


\subsection{The procedure for the definition of balancing parameters}

A procedure for determining balancing parameters has the following order: $x_{2}(t)$.

From the three times trial process we obtain three vibration records $x(t), x_{1}(t)$,

* From obtained records we calculate the maximum values, the parameters of linear approximation $\left(\xi, \xi_{1}, \xi_{2}\right)$, damping coefficient $(h)$, the particular frequency $\left(\omega_{0}\right)$, the initial forced frequency $(\omega)\left(\omega=2 \omega_{0}\right)$, the damped frequency.

* Determine the solutions of approximate systems using formulae (3.9), (3.10), (3.11).

* Calculate the maximum values $x^{\max }, x_{1}^{\max }, x_{2}^{\max }$ of the obtained solutions.

* Calculate mass $m$ and balancing location $\theta$ from formulae (3.17) and (3.18).

* Calculate $P, P_{1}, P_{2}$ from formulae (2.4), (2.5), and (2.6).

* Calculate $H=x_{\max } / P, H_{1}=x_{1 \max } / P_{1}$ and $H_{2}=x_{2 \max } / P_{2}$, and if $H \approx H_{1} \approx$ $\mathrm{H}_{2}$ then the obtained balancing parameters will have been accepted.

Example 3.3. The vibration records are given the same ones in Example 3.2 when the rotational resistant coefficient is changing. The initial conditions are chosen from the respective stationary solutions in the process creating the vibration records.

The numerical characters describing the vibration of algorithm model are given in Table 4. Assumed/Calculated imbalance parameters are shown in Table 5.

Table 5. Assumed/Calculated imbalance parameters

\begin{tabular}{|c|c|c|c|c|}
\hline \multirow{2}{*}{$\begin{array}{l}\text { Rotational resistant } \\
\text { coefficient } \\
(\alpha)\end{array}$} & \multicolumn{2}{|c|}{ Imbalance mass $m^{\prime}(\mathrm{kg})$} & \multicolumn{2}{|c|}{ Angle $\theta$ (radian) } \\
\hline & Assumed & Calculated & Assumed & Calculated \\
\hline 0.04 & 1.00000 & 1.024397 & 1.04720 & 1.058630 \\
\hline 0.06 & - & 1.004721 & - & 1.037486 \\
\hline 0.08 & - & 1.013301 & - & 1.041686 \\
\hline 0.10 & - & 0.991682 & - & 1.041103 \\
\hline 0.12 & - & 0.983110 & - & 1.065245 \\
\hline 0.14 & - & 1.009531 & - & 1.041561 \\
\hline 0.16 & - & 1.043699 & - & 1.019937 \\
\hline 0.18 & - & 1.061732 & - & 1.074192 \\
\hline 0.20 & - & 1.003184 & - & 1.049014 \\
\hline
\end{tabular}


Table 4. The numerical characters describing the vibration of algorithm model

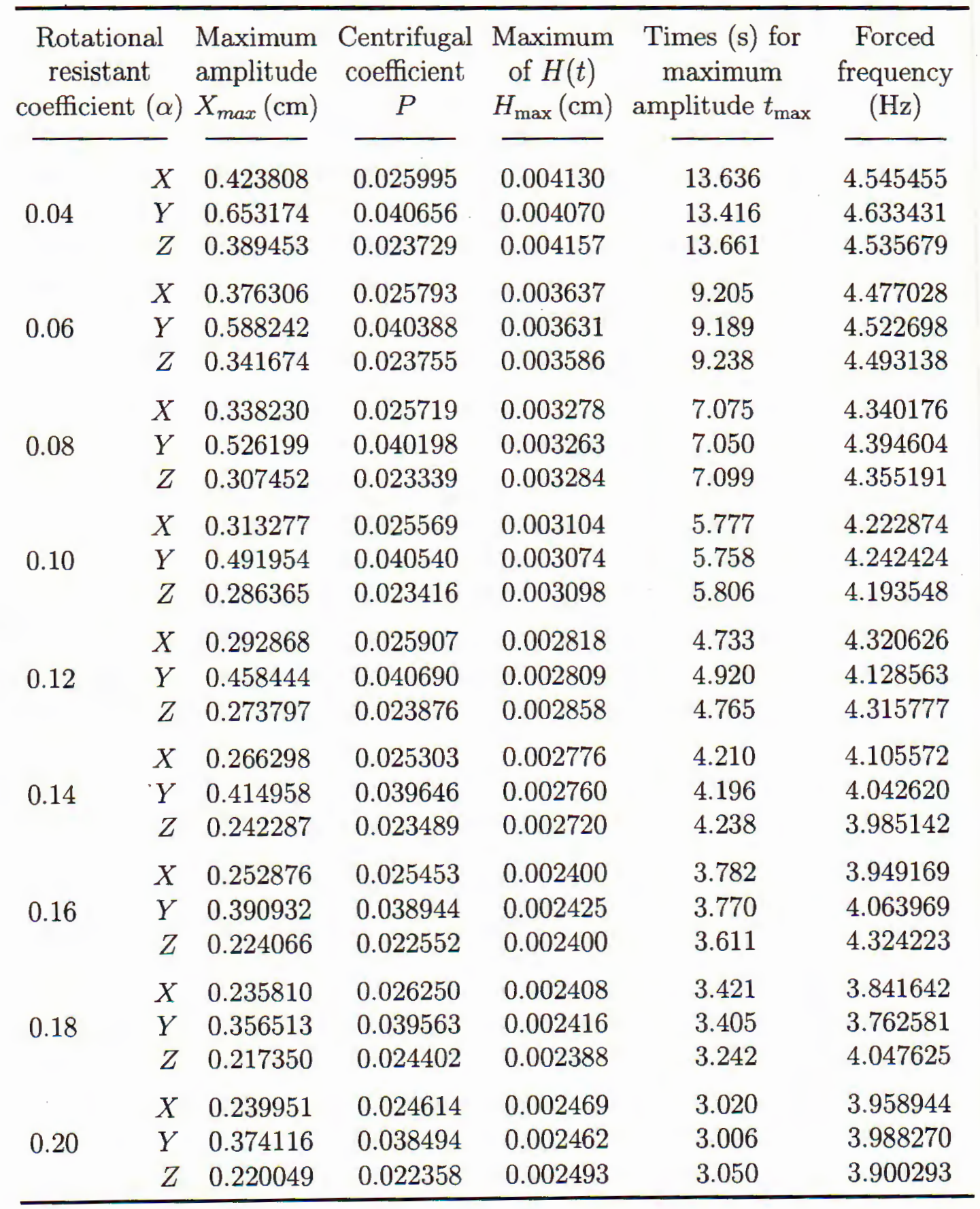

In the Table 4, column $H^{\max }$, fixing the rotational resistant coefficient $\alpha$, we show that $H^{\max } \approx H_{1}^{\max } \approx H_{2}^{\max }$. Therefore, the condition of process is satisfied.

The precise of the numerical results allows the use of the above procedure. 


\section{Conclusions}

This paper includes the following main results:

* Made the Nonlinear Differential equations of motions;

* Establish the numerical solutions for the obtained differential equations both linear and nonlinear;

* Propose a dynamical balancing method for the rotational part of machine as a nonlinear system with the decrease of rotation speed passing across a resonant region;

For the weakly nonlinear system (nonlinear parameter is small), in the status of transition, to determine the imbalance parameters, the test-three times method can be applied in accordance with above procedure.

This work is completed with financial support of the Council for Natural Science of Vietnam.

\section{REFERENCES}

1. Nguyen Cao Menh, Tran Kim Chi. Transitional Vibrations and the Balancing of Rotating Machinery; Proceeding of the National Conference "Vibrations in Engineering"; Hanoi 5-6/2000 (Vietnamese Edition).

2. Nguyen Cao Menh. The Balancing of Rotating Machinery as Nonlinear System; Vietnam Journal of Mechanics, NCSR of Vietnam Vol.21, 1999. No 3 (165-172).

3. Shabana A. A. Theory of Vibration. Volume II: Discrete and Continuous Systems. Springer-Verlag. New York Berlin London Paris Tokyo Hong Kong, Barcelona 1991.

4. Bendat J. S., Piersol A. G. Engineering Applications of Correlation and Spectral Analysis. A Wiley-Interscience Publication; John Wiley and Sons. New YorkChichester-Brisbane-Toronto, 1980.

5. The MathWorks, Inc. Using MATLAB. Version 6.

6. Nicholas J. Higham. Accuracy and Stability of Numerical Algorithms. University of Manchester, Manchester, England. Copyright 1996 by the Society for Industrial and Applied Mathematics.

7. Goloxkokob E. G., Philippov A. P. Non-Stationary Osillations of Machanical Systems. Publishing House "Science". Kiev-1966 (Russian Edition).

8. Philippov A. P. Vibrations of Machanical Systems. Publishing House "Science". Kiev-1966 (Russian Edition).

9. Timosenco X. P., Iahg D. Kh., Uiver U. Vibration Problems in Engineering. Fouth Edition, New York 1974. Publishing House "Constructive Machinery". Moscow-1985. (Russian Edition).

(xem tiếp trang 254)

Received Appril 21, 2003 